\title{
Postoperative acute kidney injury in adult non-cardiac surgery: joint consensus report of the Acute Disease Quality Initiative and PeriOperative Quality Initiative
}

John R. Prowle $\mathbb{1}^{1 凶}{ }^{\boxplus}$, Lui G. Forni $\mathbb{1}^{2,3}$, Max Bell $\mathbb{1}^{4}$, Michelle S. Chew $\mathbb{1}^{5}$, Mark Edwards ${ }^{6}$, Morgan E. Grams ${ }^{7}$, Michael P. W. Grocott ${ }^{8}$, Kathleen D. Liu $\mathbb{1 0}^{9}$, David Mcllroy ${ }^{10}$, Patrick T. Murray (1) ${ }^{11}$, Marlies Ostermann (1) ${ }^{12}$, Alexander Zarbock ${ }^{13}$, Sean M. Bagshaw ${ }^{14}$, Raquel Bartz ${ }^{15}$, Samira Bell ${ }^{16}{ }^{16}$, Azra Bihorac (1) ${ }^{17}$, Tong J. Gan ${ }^{18}$, Charles E. Hobson ${ }^{19}$, Michael Joannidis ${ }^{20}{ }^{20}$, Jay L. Koyner ${ }^{21}$, Denny Z. H. Levett ${ }^{8}$, Ravindra L. Mehta ${ }^{22}$, Timothy E. Miller ${ }^{23}$, Michael G. Mythen ${ }^{24}$, Mitra K. Nadim ${ }^{25}$, Rupert M. Pearse', Thomas Rimmele ${ }^{26}$, Claudio Ronco (1D ${ }^{27}$, Andrew D. Shaw ${ }^{28,30}$ and John A. Kellum (1) ${ }^{29,30}$

Abstract | Postoperative acute kidney injury (PO-AKI) is a common complication of major surgery that is strongly associated with short-term surgical complications and long-term adverse outcomes, including increased risk of chronic kidney disease, cardiovascular events and death. Risk factors for PO-AKI include older age and comorbid diseases such as chronic kidney disease and diabetes mellitus. PO-AKI is best defined as AKI occurring within 7 days of an operative intervention using the Kidney Disease Improving Global Outcomes (KDIGO) definition of AKI; however, additional prognostic information may be gained from detailed clinical assessment and other diagnostic investigations in the form of a focused kidney health assessment (KHA). Prevention of PO-AKI is largely based on identification of high baseline risk, monitoring and reduction of nephrotoxic insults, whereas treatment involves the application of a bundle of interventions to avoid secondary kidney injury and mitigate the severity of AKI. As PO-AKI is strongly associated with long-term adverse outcomes, some form of follow-up KHA is essential; however, the form and location of this will be dictated by the nature and severity of the AKI. In this Consensus Statement, we provide graded recommendations for $\mathrm{AKI}$ after non-cardiac surgery and highlight priorities for future research.

The development of postoperative complications after major non-cardiac surgery - particularly acute kidney injury (AKI) - has consistently been associated with substantial long-term morbidity and mortality ${ }^{1-4}$. AKI can be considered a sentinel postoperative complication that is strongly associated with increased risk of death, co-occurrence of other postoperative complications, increased length of hospital stay and the development of progressive chronic kidney disease (CKD), which results in a considerable health-care and societal burden $^{5-7}$.

Considerable research and previous consensus meetings have focused on AKI after cardiac surgery ${ }^{8}$. However, the role of AKI after other forms of major surgery, particularly gastrointestinal surgery, has received less attention, even though such procedures are associated with higher 30-day mortality than many forms of cardiac surgery ${ }^{9}$. Despite the importance of AKI as a complication after all forms of major surgery, lack of consensus exists with regard to the definition, prevention and treatment of postoperative AKI (PO-AKI) and the pathophysiology of $\mathrm{PO}-\mathrm{AKI}$ in the non-cardiac setting has not been well described. Furthermore, the understanding of and approach to this condition can differ between specialists in nephrology, anaesthesia and/or perioperative medicine.

In 2019, a joint meeting of the Acute Disease Quality Initiative (ADQI-24) and the PeriOperative 
Quality Initiative (POQI-7) was convened to address PO-AKI after major non-cardiac surgery. Here, we present our recommendations for clinical practice as well as for further research in this important area.

\section{Methods}

The Conference Chairs of the 24th ADQI/7th POQI consensus committee (J.R.P., J.A.K., A.D.S. and L.G.F.) convened a diverse panel of clinicians and researchers representing relevant disciplines (internal medicine, nephrology, critical care, anaesthesia, perioperative

\footnotetext{
Author addresses

${ }^{1}$ Critical Care and Perioperative Medicine Research Group, William Harvey Research Institute, Queen Mary University of London, London, UK.

Intensive Care Unit, Royal Surrey Hospital NHS Foundation Trust, Guildford, UK. ${ }^{3}$ Department of Clinical \& Experimental Medicine, University of Surrey, Guildford, UK. ${ }^{4}$ Department of Physiology and Pharmacology, Karolinska Institutet, Stockholm, Sweden. ${ }^{5}$ Department of Anesthesia and Intensive Care, Biomedical and Clinical Sciences, Linköping University Hospital, Linköping, Sweden.

${ }^{6}$ Anaesthesia and Critical Care Research Unit, University Hospital Southampton NHS

Foundation Trust, Southampton, UK.
}

'Division of Nephrology, Department of Medicine, Johns Hopkins University, Baltimore, MD, USA.

${ }^{8}$ Integrative Physiology and Critical Illness Group, Clinical and Experimental Sciences, Faculty of Medicine, University of Southampton, Southampton, UK.

'Divisions of Nephrology and Critical Care, Departments of Medicine and Anesthesia, University of California San Francisco School of Medicine, San Francisco, CA, USA. ${ }^{10}$ Department of Anesthesiology, Vanderbilt University Medical Center, Nashville, TN, USA.

${ }^{11}$ School of Medicine, University College Dublin, Dublin, Ireland.

${ }^{12}$ Department of Intensive Care, Guy's \& St Thomas' NHS Foundation Hospital,

London, UK.

${ }^{13}$ Department of Anesthesiology, Intensive Care and Pain Medicine, University Hospital Münster, Münster, Germany.

${ }^{14}$ Department of Critical Care Medicine, Faculty of Medicine and Dentistry, University of Alberta and Alberta Health Services, Edmonton, AB, Canada.

${ }^{15}$ Division of Critical Care Medicine, Department of Anesthesia, Duke University Medical Center, Durham, NC, USA.

${ }^{16}$ Division of Population Health and Genomics, University of Dundee, Dundee,

Scotland, UK.

${ }^{17}$ Precision and Intelligent Systems in Medicine (PrismaP), Division of Nephrology,

Hypertension, and Renal Transplantation, University of Florida, Gainesville, FL, USA.

${ }^{18}$ Department of Anesthesiology, Stony Brook University Renaissance School of Medicine, Stony Brook, NY, USA

${ }^{19}$ The PACES Center for Value in Healthcare, Framingham, MA, USA.

${ }^{20}$ Division of Intensive Care and Emergency Medicine, Department of Internal Medicine, Medical University Innsbruck, Innsbruck, Austria.

${ }^{21}$ Section of Nephrology, Department of Medicine, University of Chicago, Chicago, IL, USA.

${ }^{22}$ Division of Nephrology, Department of Medicine, University of California, San Diego, CA, USA.

${ }^{23}$ Department of Anesthesiology, Duke University School of Medicine, Durham, NC, USA.

${ }^{24}$ University College London Hospitals NIHR Biomedical Research Centre, London, UK.

${ }^{25}$ Division of Nephrology and Hypertension, Department of Medicine, Keck School

of Medicine, University of Southern California, Los Angeles, CA, USA.

${ }^{26}$ Department of Anesthesiology and Intensive Care Medicine, Edouard Herriot Hospital, Hospices Civils de Lyon, Lyon, France.

${ }^{27}$ Department of Nephrology, Dialysis and Transplantation, San Bortolo Hospital, International Renal Research Institute of Vicenza, and Department of Medicine,

University of Padova, Padova, Italy.

${ }^{28}$ Department of Anesthesiology and Pain Medicine, Alberta Health Services, Edmonton, $\mathrm{AB}$, Canada.

${ }^{29}$ Center for Critical Care Nephrology, Department of Critical Care Medicine, University of Pittsburgh, Pittsburgh, PA, USA.

${ }^{30}$ These authors contributed equally: Andrew D. Shaw, John A. Kellum. medicine, pharmacy and surgery) from Europe, North America and Australia, to discuss the issues relating to PO-AKI. The consensus meeting was held at Emmanuel College, Cambridge, UK, on 4-7 September 2019, and followed the established ADQI process and POQI protocols, including use of a modified Delphi method to achieve consensus ${ }^{8,10}$. The broad objective of ADQI 24 was to produce expert-based statements and a summary of current knowledge pertaining to the definition and management of PO-AKI in the setting of non-cardiac surgery for use by clinicians and researchers, based on review of evidence by and professional judgment of the faculty as well as to identify evidence gaps to establish research priorities.

Conference participants were divided into five work groups: group 1 was tasked with the epidemiology and pathophysiology of PO-AKI. Group 2 was tasked with developing a definition for PO-AKI. Group 3 developed recommendations for the prevention of PO-AKI. Group 4 focused on treatment options for patients with PO-AKI, and group 5 explored outcomes after PO-AKI (Supplementary Information). Members of the work groups performed comprehensive literature searches and developed a consensus of opinion, backed by evidence where possible, to distil the available literature and articulate a research agenda to address important unanswered questions. Members assessed the level of evidence and strength of recommendation for all consensus statements using the GRADE evidence to decision frameworks ${ }^{11}$. A number of ungraded research recommendations were also identified in areas where high-quality evidence is lacking. Strength of recommendations and aggregated conclusions were established by consensus of all of the participants, who formally voted and approved the consensus recommendations.

\section{Pathophysiology and epidemiology}

Like all forms of AKI, PO-AKI represents a clinical syndrome rather than a single disease and limited histological data from kidney biopsy samples are available to identify specific pathology $\mathrm{Y}^{12,13}$ (BOX 1). Animal models that are used to study AKI pathophysiology tend to employ a single insult to the kidneys rather than explore the multifactorial insults that are common in clinical practice $^{14-18}$. However, commonly implicated mechanisms for PO-AKI include ischaemia-reperfusion injury, endogenous or exogenous nephrotoxins, physical causes such as obstruction, inflammatory factors, vasoconstriction, and oxidative stress ${ }^{19}$ (FIG. 1). Thus, a variety of exposures that are encountered in the surgical setting are involved in the development of $\mathrm{PO}-\mathrm{AKI}^{20}$. Many of these exposures might also have a role in other forms of AKI, but their relative importance, severity and timing in PO-AKI may differ from that of other AKI settings. Within this framework, the contribution of individual mechanisms is often difficult to determine; for instance, nephrotoxic drugs have been implicated in $20-30 \%$ of all AKI episodes and are commonly encountered in the surgical setting ${ }^{21}$. Moreover, agents such as radiocontrast and antibiotics are commonly used in patients who are already at risk of AKI and discerning their relative contribution to the overall AKI course is 
Box 1 | Pathophysiology and epidemiology of PO-AKI

\section{Consensus Statement 1a}

Postoperative acute kidney injury (PO-AKI) is a clinical syndrome rather than a single disease. The majority of cases of PO-AKI are multifactorial in cause (ungraded).

\section{Consensus Statement 1b}

The incidence of PO-AKI (defined by changes in serum creatinine) varies with the characteristics and urgency of surgery. The incidence of AKI after day or ambulatory surgery is uncertain (ungraded).

\section{Consensus Statement 1c}

AKI defined by transient oliguria is more common in the intraoperative and postoperative period than AKI defined by serum creatinine. Severe oliguria and anuria, even in the absence of changes in serum creatinine, are associated with increased long-term morbidity and mortality (ungraded).

\section{Consensus Statement 1d}

The majority of observational studies focus on AKI in the immediate postoperative period. Little evidence is available regarding the epidemiology of acute kidney disease in the perioperative setting (ungraded).

\section{Consensus Statement 1e}

Risk factors for $\mathrm{PO}-\mathrm{AKI}$ include age $>50$ years, male sex, glomerular filtration rate $<60 \mathrm{ml} / \mathrm{min} / 1.73 \mathrm{~m}^{2}$, diabetes mellitus, heart failure, ascites, hypertension, emergency surgery, intraperitoneal surgery, number of medications, use of angiotensin-converting enzyme (ACE) inhibitors or angiotensin receptor blockers (ARBs), high American

Society of Anesthesiology Physical Status classification score and albuminuria. Patients with chronic kidney disease (CKD) and/or diabetes are at a particularly high risk of AKI (ungraded). concentration of tissue inhibitor of metalloproteinases 2 (TIMP2) multiplied by that of insulin-like growth factor binding protein 7 (IGFBP7) added prognostic information to AKI defined by urine output alone ([TIMP2] $\times[$ IGFBP7] $>2$ was associated with progression from stage $1 \mathrm{AKI})^{36}$.

Many, but not all, of the available studies suggest that PO-AKI defined by oliguria alone is more common than PO-AKI defined by changes in serum creatinine levels ${ }^{26,37-39}$. In a study of PO-AKI after non-cardiac surgery, the incidence of AKI increased from $8 \%$ to $64 \%$ if urine output was included in the definition ${ }^{37}$. Some postoperative oliguria is assumed to be an appropriate physiological response to decreased intravascular volume, vasodilatation or non-osmotic release of arginine vasopressin in response to tissue injury ${ }^{40}$; however, sustained decrements in urine output, even without accompanying changes in serum creatinine, have been associated with longer-term morbidity and mortality ${ }^{38,41}$.

\section{Epidemiology of postoperative AKD}

The concept of acute kidney disease (AKD) is intended to bridge the gap between AKI, which refers to an acute alteration in kidney function over a 7-day period, and $\mathrm{CKD}$, which refers to a sustained alteration in kidney function over a 3-month period. AKD therefore refers to AKI that persists for more than 7 days after the initial insult, or a progressive decline in kidney function that does not meet AKI criteria on a week-by-week basis. Few epidemiological data are available on the incidence and prognosis of postoperative $\mathrm{AKD}$, despite its likely importance as a bridge to development of sustained kidney dysfunction and $\mathrm{CKD}^{42}$. A study that evaluated substantial decline in kidney function, defined as a $30 \%$ decrease in estimated glomerular filtration rate at 60 days after surgery, suggested that the incidence of this outcome was only $2 \%$ among patients without PO-AKI (defined by change in serum creatinine only), but $10 \%, 17 \%$ and $29 \%$ among patients who experienced PO-AKI stages 1, 2, and 3 , respectively ${ }^{43}$. The incidence of AKD in the general population is likely lower than in the post-surgery population; in a study of Canadian electronic health records, $5.2 \%$ of patients in a given period had AKD with or without $\mathrm{AKI}^{44}$.

Risk factors for postoperative AKI. Several patientrelated, surgery-related, and anaesthetic-related risk factors for developing early PO-AKI during the first $48 \mathrm{~h}$ after surgery have been described ${ }^{4,40}$. The three validated risk scores for PO-AKI include male sex, age $>50$ years, diabetes mellitus, hypertension, ascites, heart failure, emergent surgery, intraperitoneal surgery, polypharmacy, use of an angiotensin-converting enzyme (ACE) inhibitor or angiotensin receptor blocker (ARB), and increasing American Society of Anesthesiologists physical status classification score $\mathrm{s}^{34,45,46}$. Albuminuria and hypoalbuminaemia have also been described as risk factors for PO-AKI ${ }^{47-50}$. Risk factors for PO-AKI occurring in patients in the ICU more than $48 \mathrm{~h}$ after surgery reflect risk factors for AKI in critical illness including the need for new nephrotoxic medication, sepsis and shock $^{51,52}$. Whether targeting modifiable risk factors for 


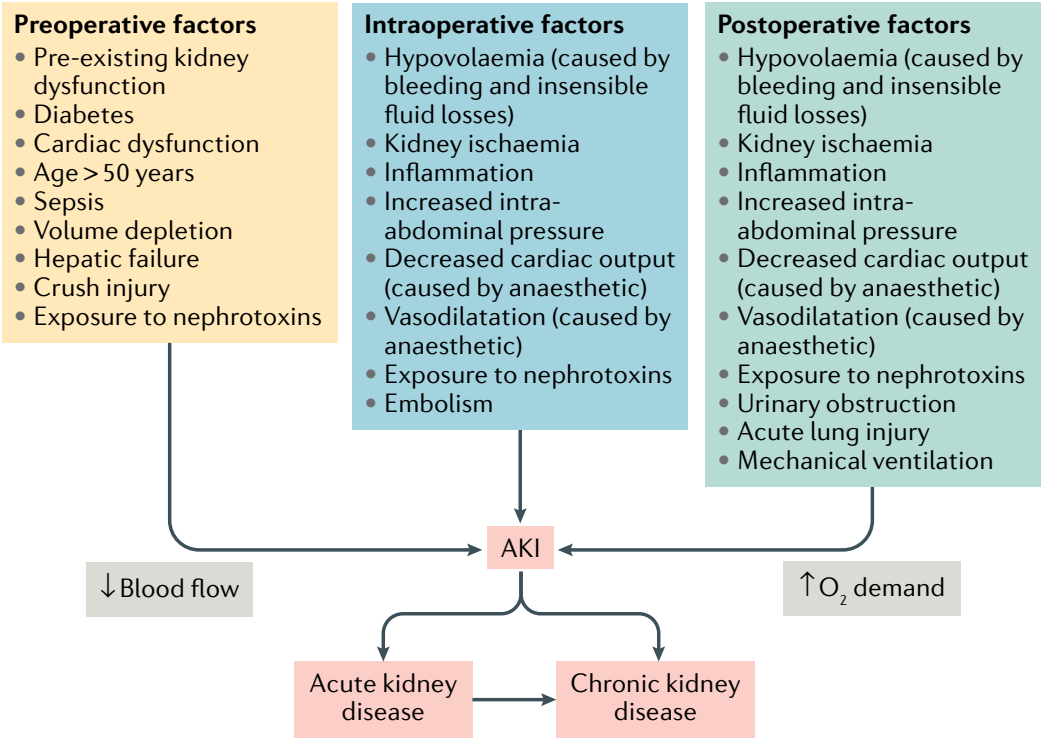

Fig. 1 | Pathophysiology of PO-AKI. Similar to most other forms of acute kidney injury (AKI), postoperative AKI (PO-AKI) commonly has a multifactorial aetiology, which is mediated by common injury pathways that affect the kidney microcirculation, oxygen demand and inflammation. In most cases, a combination of preoperative risk factors, intraoperative events and postoperative events leads to the development of AKI. Baseline risk factors and the persistence and severity of injurious factors in the postoperative setting also determine the outcomes of AKI, acute kidney disease and eventually chronic kidney disease. Adapted from Acute Disease Quality Initiative 24, www.ADQI.org, CC BY 2.0 (https://creativecommons.org/licenses/by/2.0/).

early PO-AKI, for example, using perioperative haemodynamic interventions, will impact the incidence of AKI later in the postoperative period, $>48 \mathrm{~h}$ after surgery, remains uncertain.

Use of a validated clinical risk score is a potentially useful approach to risk stratify patients for targeted interventions or to facilitate the conduct of randomized controlled trials (RCTs) ${ }^{53}$. However, existing validated risk scores for PO-AKI were developed using a variety of older (pre-KDIGO) definitions of AKI or were applied to select surgical settings $\mathrm{s}^{34,45,46}$. More generalizable prognostic clinical tools are needed to accurately risk-stratify patients preoperatively, particularly to distinguish those patients at the highest levels of risk (e.g. $>20-30 \%)$. In the absence of such tools, patients with $\mathrm{CKD}$ and those with diabetes can reasonably be considered to be at increased risk of PO-AKI. The KDIGO guideline for the diagnosis, evaluation and management of AKI suggests that age $>60$ years, emergency surgery, elevated American Society of Anesthesiologists physical status classification score and preoperative comorbid illnesses, including diabetes and any chronic disease of the heart, lung or liver, are risk factors for PO-AKI ${ }^{42}$.

Research recommendations. Understanding the pathophysiology and epidemiology of PO-AKI is central to the identification and development of novel preventative therapies and treatments. This understanding may be improved by conducting prospective studies that include collection of biological samples for histological and biomarker examination as well as retrospective analyses that examine shared pathogenesis across a spectrum of perioperative complications. The incidence of PO-AKI in the day and ambulatory surgery settings is uncertain and should be investigated in epidemiological studies. Development of better risk prediction tools for PO-AKI could help to risk-stratify patients and set thresholds of predicted risk that merit adjustments in perioperative care.

\section{Definition of postoperative AKI}

Although the KDIGO definition of AKI is now widely employed in the perioperative literature, the exact implementation of this definition varies considerably in terms of timeframe of diagnosis. We recommend defining $\mathrm{PO}-\mathrm{AKI}$ as occurring when existing KDIGO criteria for AKI are met within 7 days of an operative intervention (BOX 2). AKI occurring de novo $\geq 7$ days after surgery may arise in a variety of contexts that are not necessarily related to the surgery itself; we recommend that such AKI should be evaluated and managed as hospital-acquired AKI. Our recommendation for using a 7-day window is somewhat arbitrary but maintains important consistency with the KDIGO definitions for AKI in other clinical contexts ${ }^{54}$ and is supported by evidence from numerous clinical studies ${ }^{51,55-57}$. Furthermore, within the 7-day window, different timing of AKI may be indicative of the nature of the kidney insult, that is, intra-operative events versus post-operative insults.

Notably, AKI could potentially be present before surgery (for example, in patients with sepsis requiring surgical intervention, acute trauma or preoperative contrast exposure), highlighting the importance of a risk-based kidney health assessment (KHA) ${ }^{51}$. Use of a KHA was previously recommended by $\mathrm{ADQI}$ in the context of quality improvement processes for monitoring patients at a high risk of AKI before surgery ${ }^{58}$. A KHA comprises a structured evaluation that includes previous history of AKI, current medications, cardiovascular health, haemodynamic status and markers of kidney function (i.e. serum creatinine) and kidney damage (i.e. urine dipstick). Use of a KHA in the perioperative period involves integrated assessment of patient-specific and procedure-specific risk factors, clinical context and resource setting.

Importantly, the KHA approach can be used to perform context-dependent evaluation both before and after surgery, when AKI is diagnosed and during follow-up (FIG. 2). A perioperative KHA may range from a simple history and physical examination conducted as part of a routine low-risk preoperative evaluation, to an evaluation that includes more specific measures such as urinalysis to identify proteinuria, biomarkers of kidney damage and kidney imaging in higher-risk patients (BOX 2). Measurements other than serum creatinine and urine output are not currently part of the KDIGO AKI definition, but likely provide important context for enhanced interpretation of these criteria. Although we strongly support routine use of KHAs before and after surgery, the potential clinical benefit of this approach has not been formally evaluated and so remains uncertain. However, an accepted advantage of routine perioperative KHAs is improved recognition of preoperative kidney dysfunction, which establishes a baseline for subsequent diagnosis of PO-AKI. 
Conventional diagnostic criteria for AKI might be influenced by multiple factors specific to the perioperative period, rendering their predictive utility for PO-AKI less certain. Serum creatinine concentration is subject to abrupt changes in total body water ${ }^{59}$, which may spuriously increase or decrease measured values, as well as perioperative changes in creatinine production that alter serum creatinine levels independently of changes in $\mathrm{GFR}^{60}$. Urine output is also likely to be influenced by multiple physiological factors specific to the perioperative period, including intravascular volume status, relative or absolute hypotension and neurohormonal response to surgery. Data suggest that although intraoperative oliguria is associated with PO-AKI, the positive predictive value is low $^{32}$. We therefore recommend that urine output is interpreted in the context of severity of oliguria, clinical setting and other corroborating evidence of PO-AKI. Thus, isolated perioperative oliguria meeting AKI stage 1 criteria in the context of transient hypotension should not be viewed as being the same as more severe or prolonged oligo-anuria unresponsive to haemodynamic interventions. The presence of new evidence of kidney tubular damage (e.g. on urine microscopy or from novel biomarkers) or evidence of evolving sepsis in the context of early oliguria would be suggestive of AKI; these factors can be identified and integrated within a timely postoperative KHA.

Definition of postoperative AKD. Consistent with the definition of AKD as a condition in which AKI stage 1 or greater is present $\geq 7$ days after an AKI initiating event, postoperative acute kidney disease (PO-AKD) occurs in patients with $\mathrm{PO}-\mathrm{AKI}$ who continue to meet KDIGO criteria for AKI $>7$ days after surgery. Patients whose rise in serum creatinine level begins before postoperative day 7 but whose serum creatinine level does not increase $\geq 50 \%$ above baseline until 7-90 days after surgery would also meet PO-AKD criteria based on a slow progressive deterioration in kidney function in the postoperative period ( $\mathrm{BOX} 2$ ). However, the greater the time interval between surgery and identification of kidney injury the less likely that the injury could be attributed to the perioperative process. Patients who meet criteria for AKI for the first time after postoperative day 7 without any previous increase in serum creatinine should therefore be referred to as having $\mathrm{AKI}^{54}$ or $\mathrm{AKD}^{61}$ without the

\section{Box 2 | Definition of PO-AKI}

\section{Consensus Statement 1a}

We recommend defining postoperative acute kidney injury (PO-AKI) as occurring when existing Kidney Disease Improving Global Outcomes (KDIGO) criteria for AKI are met within 7 days of an operative intervention (ungraded).

\section{Consensus Statement 1b}

We recommend performing a risk-based Kidney Health Assessment (KHA) preoperatively and postoperatively, with the specific methods and frequency of assessment determined by clinical judgement based on individual patient-specific and procedural-specific risk factors and the available resources (grade $D$ evidence; strong recommendation).

\section{Consensus Statement 1c}

We recommend using the term postoperative acute kidney disease (PO-AKD) when $\mathrm{KDIGO}$ criteria for $\mathrm{AKI}$ are present after postoperative day 7 and objective evidence of injury initiation was present within the first 7 postoperative days (ungraded). postoperative prefix. With this proposed nomenclature (FIG. 3), it sought to combine a level of consistency with existing recommendations for use of $\mathrm{AKI}$ and $\mathrm{AKD}$ terminology ${ }^{54,61}$. However, revision may be warranted as our understanding of the pathophysiology and prognostic implications of temporal variations in the postoperative disease course evolves.

Research recommendations. Limited information exists regarding the prognostic implications of the nature and timing of the AKI diagnosis within 7 days after surgery, and these important aspects of the PO-AKI diagnostic criteria warrant better characterization. Furthermore, to what extent indices of kidney injury other than serum creatinine and urine output, such as urinary or plasma biomarkers, are important for prognostication in PO-AKI is unknown and should be the subject of further study. Whether or not important differences exist in the epidemiology and prognostic significance of PO-AKD that arises as persistent PO-AKI compared with $\mathrm{PO}-\mathrm{AKD}$ that develops after a gradual rise in creatinine after day 7 is also unknown.

The impact of routine, structured KHA on the detection of PO-AKI remains to be determined. An expanded perioperative urinalysis conducted as part of a detailed KHA might identify previously unknown pathology and could potentially lead to additional, potentially expensive and/or invasive investigations that could delay surgery. The presumed benefits and cost-effectiveness of a KHA therefore require confirmation. Finally, the optimal interpretation of intraoperative and postoperative oliguria remains unclear and should be the subject of further study.

\section{Prevention of postoperative AKI}

Preoperative strategies. Surgery is sometimes performed in patients with established AKI; however, common perioperative interventions focus on prevention of PO-AKI (BOX 3). As nephrotoxic drugs are an important preventable contributor to the risk of PO-AKI, exposure to these drugs should be limited whenever possible and the benefits of the intervention weighed against the risk of developing or worsening $\mathrm{AKI}^{62}$. Although the avoidance of potential nephrotoxins is considered to be a cornerstone of AKI prevention, there is a relative paucity of data supporting this approach. However, routine use of gentamicin for surgical prophylaxis was associated with an increased risk of AKI following orthopaedic surger $y^{63}$ and, in the paediatric setting, systematic screening for nephrotoxin use, with a focus on close monitoring in patients exposed to multiple nephrotoxins and nephrotoxin cessation, has been shown to reduce rates of $\mathrm{AKI}^{64,65}$.

At present, common practice is to discontinue use of ACE inhibitors and ARBs before surgery to avoid perioperative hypotension. This approach is assumed to reduce the risk of perioperative AKI based on physiological rationale. In line with this established practice, we recommend discontinuing ACE inhibitors and ARBs for a minimum of $24 \mathrm{~h}$ before surgery to minimize the risks of perioperative hypotension and/or postoperative AKI (BOX 3). This recommendation is weak owing to limited data in support of this practice and is likely 


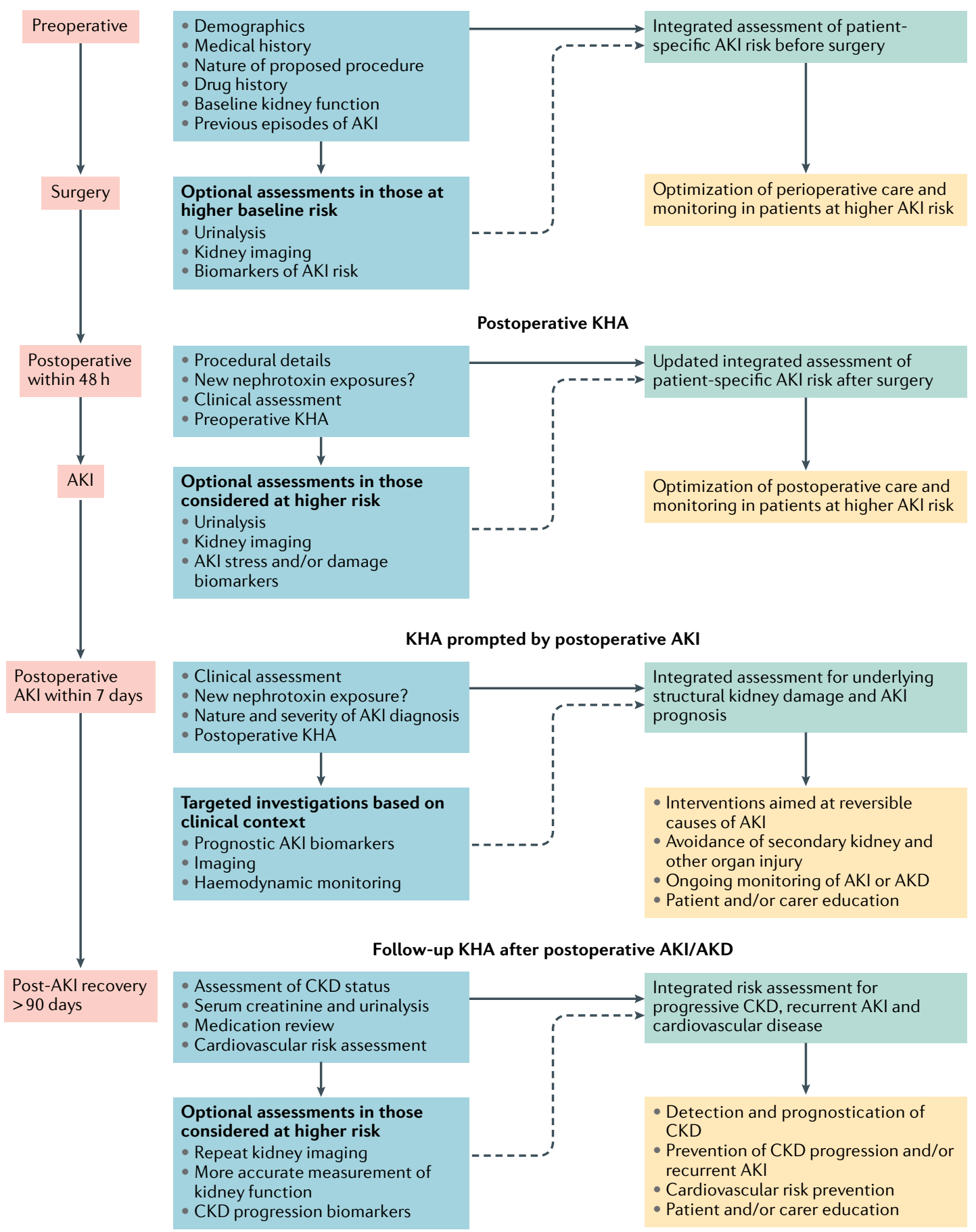

Fig. 2 | The role of the KHA in postoperative AKI. Kidney health assessments (KHAs) can be used in the risk assessment, detection, management and follow-up of postoperative acute kidney injury (AKI). A series of context-specific KHAs involving integration of medical history and clinical context, potentially in combination with further investigations, such as analysis of specific kidney biomarkers or imaging, in higher-risk settings, can provide kidney prognostic information to guide further monitoring and treatment. AKD, acute kidney disease; CKD, chronic kidney disease. Adapted from Acute Disease Quality Initiative 24, www.ADQI.org, CC BY 2.0 (https://creativecommons.org/licenses/by/2.0/).

to be revised as more evidence becomes available. For instance, an analysis of 949 patients undergoing major gastrointestinal or hepatobiliary surgery failed to show a difference in the rates of AKI between those who did and those who did not have ACE inhibitors and ARBs withheld before surgery ${ }^{66}$.
NSAIDs are generally avoided in surgical patients at a high risk of AKI, but might be beneficial as opiatesparing agents in patients who are at a low risk of AKI. Although NSAIDs are widely used in patients with normal kidney function, their impact on risk of AKI in the postoperative period is unclear ${ }^{24}$. 


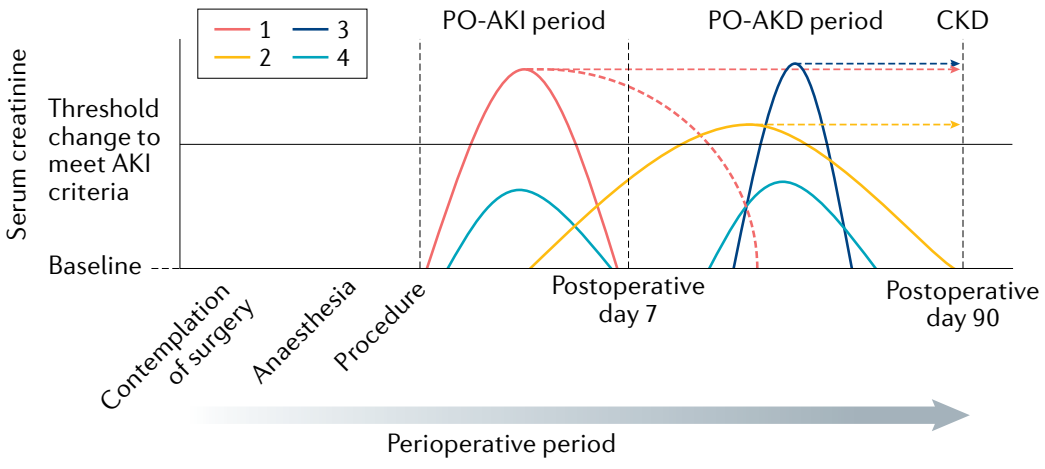

Fig. 3 | Conceptual model of PO-AKI and PO-AKD. Postoperative acute kidney injury (PO-AKI) occurs when the Kidney Disease Improving Global Outcomes (KDIGO) criteria for $\mathrm{AKI}$ are met within 7 days of an operative intervention. Postoperative acute kidney disease (PO-AKD) occurs when patients with PO-AKI continue to meet KDIGO AKI criteria $\geq 7$ days after surgery or when patients whose serum creatinine levels began to rise following surgery meet KDIGO AKI criteria $\geq 7$ days after surgery. A number of potential trajectories of serum creatinine are depicted with suggested application of the proposed nomenclature. $\mathrm{PO}-\mathrm{AKI}$ might commence and resolve before postoperative day 7 or persist after postoperative day 7 and therefore be classed as PO-AKD. If PO-AKD continues after postoperative day 90 it will be classed as chronic kidney disease (CKD) (trajectory 1). PO-AKD also occurs when evidence of new kidney injury was present before postoperative day 7 but did not meet the criteria for PO-AKI until after postoperative day 7. This form of PO-AKD might also either recover before postoperative day 90 or continue after postoperative day 90 and be classed as CKD (trajectory 2). Stand-alone $\mathrm{AKI}$ or $\mathrm{AKD}$ (that is, $\mathrm{AKI}$ or AKD that is seemingly not related to the operative intervention) can also occur during the perioperative period. As these new events occur distant to the surgical insult they should not be referred to as PO-AKI or PO-AKD and should be considered in the context of their direct precipitants (trajectory 3). Subclinical kidney injury can occur before or after postoperative day 7 (trajectory 4). This subclinical injury does not meet current criteria for AKI or AKD, but may be identified by risk-based serial kidney health assessments (KHAs). Source: Adapted from Acute Disease Quality Initiative 24, www.ADQI.org, CC BY 2.0 (https://creativecommons.org/licenses/by/2.0/).

To date, no preoperative pharmacological intervention has been shown to reduce the risk of PO-AKI in RCTs. However, attention should be paid to ensuring euvolaemia prior to surgery and addressing or avoiding preoperative fluid losses caused by excessive fasting, bowel preparation and/or acute illness ${ }^{67}$.

\section{Box 3 | Prevention of PO-AKI: preoperative strategies}

\section{Consensus Statement 3a}

We recommend that when considering use of medications or interventions that are associated with an increased risk of acute kidney injury (AKI), the relative benefits and/or necessity of the medications or interventions should be balanced with the risk of postoperative AKI (PO-AKI). This recommendation applies to outpatient medications, new medications and exposures including contrast media (grade $\mathrm{C}$ evidence; strong recommendation).

\section{Consensus Statement 3b}

We recommend discontinuing angiotensin-converting enzyme (ACE) inhibitors and angiotensin receptor blockers (ARBs) for a minimum of $24 \mathrm{~h}$ before surgery, dependent on the specific medication, to minimize the risks of perioperative hypotension and/or PO-AKI (grade D evidence, weak recommendation).

\section{Consensus Statement 3c}

We recommend assessing the relative risks and benefits of use of NSAIDs (including COX2 inhibitors) in individual patients. In general, in patients at a high risk of PO-AKI, the risk of AKI associated with use of NSAIDs might outweigh the benefits with regard to opioid minimization, whereas in low-risk patients, the benefits of use of NSAIDs might outweigh the risks. The risk-benefit profile in patients at a moderate risk of AKI is particularly uncertain (grade $D$ evidence; strong recommendation).
Intraoperative strategies. Intravenous fluid and haemodynamic management in the intraoperative and early postoperative period has a major impact on the development of PO-AKI (BOX 4). The RELIEF RCT in 3,000 patients undergoing major elective non-cardiac surgery compared a zero fluid balance target with a "moderately liberal" approach to fluid administration and demonstrated an increased risk of AKI in the restrictive, zero fluid balance group ${ }^{68}$. Algorithms that use cardiac output monitoring to optimize cardiac stroke volume and/or increase global oxygen delivery, known as goal-directed therapy, have been the subject of multiple RCTs. A Cochrane review and meta-analysis suggested that goal-directed therapy can reduce the risk of PO-AKI ${ }^{69,70}$. Large observational studies have shown strong associations between intraoperative hypotension and PO-AKI with the risk of organ injury a function of the severity and duration of the hypotension ${ }^{71-73}$. Both absolute mean arterial blood pressure (MAP (e.g. 60-70 mmHg)) and relative MAP (e.g. $<30 \%$ reduction from baseline) thresholds are associated with $\mathrm{PO}-$ AKI. The results of an interventional trial of use of a MAP target of $>65 \mathrm{mmHg}$ as part of a goal-directed strategy have added weight to these observational data, demonstrating fewer postoperative complications (including AKI) and shorter length of hospital stay with this approach ${ }^{74}$. Similar to other settings such as sepsis, a higher MAP target may be beneficial in reducing the incidence of AKI in patients with poorly controlled pre-existing hypertension ${ }^{75}$.

Fluid composition may also affect the risk of PO-AKI; $0.9 \%$ saline is associated with increased risk of PO-AKI compared with balanced crystalloids ${ }^{76-78}$ (BOX 4). High-quality evidence has shown that use of starchbased colloids increases the risk of AKI and other adverse outcomes in patients with sepsis and other critically ill patients ${ }^{79,80}$. Although caution is advised when using starch-based colloids, the limited data in the elective perioperative setting, where lower fluid volumes may be given than in treatment of septic shock, suggest little difference in kidney outcomes when starch-based colloids are used in preference to crystalloids or non-starch colloids ${ }^{81,82}$. Ongoing research is investigating use of starch-based colloids versus crystalloids in the perioperative setting. One such trial published in 2020 reported no significant difference in a composite outcome of death or postoperative complications within 14 days of major abdominal surgery between patients who received low-molecular-weight hydroxyethyl starch compared with those who received $0.9 \%$ saline $^{83}$.

Postoperative strategies. The early postoperative period has not been extensively studied in relation to kidney outcomes. However, therapeutic strategies used in the hours after surgery could likely modify the risk of AKI (BOX 5). Clinically important postoperative hypotension (defined as systolic blood pressure $<90 \mathrm{mmHg}$ and requiring medical intervention) affects over $30 \%$ of patients, often goes uncorrected for longer than intraoperative hypotension and is associated with an increased risk of all-cause mortality and myocardial injury ${ }^{84}$. These findings suggest that maintenance of fluid and 


\section{Box 4 | Prevention of PO-AKI: intraoperative strategies}

\section{Consensus Statement 4a}

We recommend not using restrictive or zero-balance perioperative fluid regimens in major elective surgery (except in specific circumstances). Such regimens are associated with increased risk of postoperative acute kidney injury (PO-AKI) (grade B evidence; strong recommendation).

\section{Consensus Statement 4b}

We recommend the use of goal-directed haemodynamic therapy in high-risk patients to optimize volume status, blood pressure and cardiac output and reduce the risk of PO-AKI (grade B evidence; strong recommendation).

\section{Consensus Statement 4c}

We recommend maintaining an intraoperative mean arterial blood pressure (MAP) $>65 \mathrm{mmHg}$ to reduce the risk of PO-AKI. The risk of AKI is a function of both the magnitude of hypotension and its duration. For selected patients, including those with pre-existing systemic hypertension, a higher MAP target should be considered (grade $\mathrm{C}$ evidence; weak recommendation).

\section{Consensus Statement 4d}

We recommend the perioperative use of balanced crystalloids rather than $0.9 \%$ saline to reduce the risk of $\mathrm{PO}-\mathrm{AKI}$ (grade $\mathrm{C}$ evidence; strong recommendation).

\section{Consensus Statement 4e}

In the setting of elective surgery outside of the ICU, insufficient evidence exists to make a recommendation regarding the choice of crystalloids or colloids for volume expansion to modify the risk of PO-AKI (ungraded).

haemodynamic status in the early postoperative phase is important to avoid organ injuries including $\mathrm{AKI}^{28}$. A trial that used stroke volume optimization and vasopressors to target a MAP within $10 \%$ of the preoperative baseline value during and for $4 \mathrm{~h}$ after surgery showed that this strategy was associated with a reduction in postoperative organ injury, but was not powered to assess kidney-specific outcomes ${ }^{85}$. Further research is warranted to evaluate postoperative monitoring strategies and ideal clinical settings to reduce the risk of PO-AKI in moderate- to high-risk patients.

As postoperative hyperglycaemia is strongly associated with AKI, avoidance of perioperative hyperglycaemia

\section{Box 5 | Prevention of PO-AKI: postoperative strategies}

\section{Consensus Statement 5a}

We recommend maintenance of adequate organ perfusion, including attention to euvolaemia and treatment of hypotension in the early postoperative period, to reduce the risk of postoperative acute kidney injury (PO-AKI). Monitoring and targeting haemodynamic parameters may require enhanced levels of monitoring and/or care depending on the clinical context and patient risk profile (grade D evidence; strong recommendation).

\section{Consensus Statement $\mathbf{5 b}$}

We recommend treatment of postoperative hyperglycaemia (target glucose $<180 \mathrm{mg} / \mathrm{dl}$ $[<10 \mathrm{mmol} / \mathrm{l}]$ ) to reduce the risk of $\mathrm{PO}-\mathrm{AKI}$ (grade $\mathrm{C}$ evidence; strong recommendation).

\section{Consensus Statement 5c}

We recommend considering restarting angiotensin-converting enzyme (ACE) inhibitor or angiotensin receptor blocker (ARB) therapy within the first $48 \mathrm{~h}$ postoperatively in patients who are haemodynamically stable without evidence of PO-AKI. This decision should be individualized and context-specific (grade $\mathrm{C}$ evidence; strong recommendation).

\section{Consensus Statement 5d}

We recommend that enhanced recovery after surgery (ERAS) pathways (for example, use of NSAIDs and avoiding markedly positive fluid balance) are individualized based on the baseline risk of PO-AKI. The impact of ERAS pathways on the incidence of PO-AKI is unclear owing to the potential for both beneficial and harmful effects (grade $\mathrm{C}$ evidence; strong recommendation).
$(>180 \mathrm{mg} / \mathrm{dl})$ is recommended ${ }^{86}$. Failure to restart chronic ACE inhibitor or ARB therapy that was suspended preoperatively is associated with increased 30 -day mortality ${ }^{87,88}$. However, the optimal time point to restart these medications has not been clearly established. Given their effects on the kidney, a reasonable approach would be to conduct a focused KHA before restarting ACE inhibitors or ARBs.

Enhanced recovery after surgery pathways are typically multimodal, multidisciplinary interventions that have been promoted to facilitate recovery from surgery. Use of NSAIDs to minimize opiate analgesia requirements is a common component of such pathways, along with avoidance of markedly positive fluid balance. Accordingly, in some settings such as colorectal surgery, implementation of an enhanced recovery after surgery pathway could be associated with a higher, rather than lower, incidence of $\mathrm{AKI}^{89}$.

\section{Treatment of postoperative AKI}

As a general principal, treatment of AKI should be initiated as early as possible, including in patients with suspected AKI or who are at a substantially increased risk of AKI. Management of PO-AKI involves specific approaches, for example, avoidance of nephrotoxins, as well as general management principles that are common to all postoperative complications, such as haemodynamic optimization. Similarly, treatment of PO-AKI shares many features with the treatment of AKI in other settings (TABLE 1). Treatment goals include reducing kidney injury and complications related to decreased kidney function.

A full KHA should be performed, including drug history, which should focus on potential nephrotoxins, including antibiotics and radiocontrast agents. In addition to classical evaluation techniques, a number of biomarkers of cellular damage and functional change are available for the early diagnosis, risk assessment and prognosis of $\mathrm{AKI}^{90-95}$. Nephrology or urology consultation should be sought if intrinsic causes of AKI or obstruction are suspected based on the KHA results. The frequency and duration of monitoring should be individualized based on patient risk, exposure and clinical course (BOX 6).

The KDIGO bundle. The treatment and prevention bundle outlined in the 2012 KDIGO clinical practice guideline for AKI consists of supportive measures, including volume management, maintenance of adequate blood pressure and judicious avoidance of nephrotoxins. Two RCTs demonstrated that implementation of this bundle in high-risk patients identified by biomarkers after surgery significantly reduced the occurrence of PO-AKI, but were not powered to demonstrate differences in longer-term patient outcomes ${ }^{96,97}$. However, observational studies have shown that the severity of AKI correlates with short-term ${ }^{98-101}$ and longer-term ${ }^{102}$ adverse events, suggesting that interventions that reduce the severity of AKI could improve these outcomes.

Kidney replacement therapy. Kidney replacement therapy (KRT) may be required in patients with severe AKI who develop medically refractory fluid and/or metabolic 
Table 1 | Adaptation of the KDIGO guidelines for treatment of AKI to the postoperative setting

\begin{tabular}{|c|c|c|}
\hline ADQI-POQI recommendations ${ }^{\mathrm{a}}$ & $\begin{array}{l}\text { KDIGO strength of } \\
\text { recommendation }\end{array}$ & $\begin{array}{l}\text { KDIGO grade } \\
\text { of evidence }\end{array}$ \\
\hline $\begin{array}{l}\text { In the absence of haemorrhagic shock, we suggest using a balanced and } \\
\text { buffered isotonic crystalloid (e.g. Ringer's lactate) rather than colloids } \\
\text { (albumin or starches) as initial management for expansion of intravascular } \\
\text { volume in patients with PO-AKI }\end{array}$ & Strong & B \\
\hline $\begin{array}{l}\text { We recommend the use of vasopressors in conjunction with fluids } \\
\text { in patients with vasomotor shock with PO-AKI }\end{array}$ & Strong & $\mathrm{D}$ \\
\hline $\begin{array}{l}\text { We suggest using protocol-based management of haemodynamic and } \\
\text { oxygenation parameters to treat patients with PO-AKI and to prevent } \\
\text { worsening of AKI in high-risk patients in the perioperative setting }\end{array}$ & Strong & $\mathrm{D}$ \\
\hline $\begin{array}{l}\text { We suggest insulin therapy targeting plasma glucose }<180 \mathrm{mg} / \mathrm{dl}(10 \mathrm{mmol}) \\
\text { in patients with PO-AKI }\end{array}$ & Weak & Not graded \\
\hline $\begin{array}{l}\text { We suggest not using diuretics to treat AKI, except in the management } \\
\text { of volume overload }\end{array}$ & Strong & A \\
\hline $\begin{array}{l}\text { We recommend not using low-dose dopamine fenoldopam, atrial } \\
\text { natriuretic peptide or recombinant human IGF1 to treat AKI }\end{array}$ & Strong & A \\
\hline $\begin{array}{l}\text { We recommend not using nephrotoxic drugs in patients with PO-AKI unless } \\
\text { no suitable, less nephrotoxic alternatives are available or the benefits } \\
\text { outweigh the risks }\end{array}$ & Strong & A \\
\hline
\end{tabular}

ADQI, Acute Disease Quality Initiative; AKI, acute kidney injury; IGF1, insulin-like growth factor I. KDIGO, Kidney Disease Improving Global Outcomes; PO-AKI, postoperative AKI; POQI, PeriOperative Quality Initiative. ${ }^{a}$ Adapted from the treatment recommendations in the $2012 \mathrm{KDIGO}$ clinical practice guideline for $\mathrm{AKI}^{54}$.

complications. In the absence of conventional indications, the best timing for initiating KRT in PO-AKI is uncertain. Potential rationales for initiating KRT during PO-AKI are avoidance of fluid accumulation, maintenance of acid-base and electrolyte control, provision of space to accommodate nutritional and medication needs, and abrogation of the interaction between the kidney and other organs. Theoretically, early initiation of KRT could facilitate better fluid, electrolyte and acid-base homeostasis. However, KRT is associated with increased risk of complications, healthcare costs and clinical workload.

The 2012 KDIGO guidelines recommend starting KRT "when life threatening changes in fluid, electrolyte, and acid-base balance exist" and considering the "broader clinical context, the presence of conditions that can be modified with [KRT], and trends of laboratory tests - rather than single BUN and creatinine thresholds alone - when making the decision to start [KRT]". Contradictory trial results ${ }^{103,104}$ have stimulated debate regarding the best timing for KRT in patients with established AKI and differences in study design, patient populations, definitions of early and late RRT initiation, and inclusion criteria make head-to-head comparisons difficult.

Following our consensus conference, the results of the STARRT-AKI study provided high-quality evidence that early commencement of KRT based on AKI criteria in the absence of conventional indications does not improve survival, may needlessly expose some patients to KRT and results in a higher risk of KRT dependence at 90 days compared with a conservative strategy for starting $\mathrm{KRT}^{105}$. Importantly, STARRT-AKI included a large cohort of patients with PO-AKI $(n=965)$ and showed no evidence of a benefit of early commencement of KRT in this subgroup (odds ratio for mortality with earlier KRT: 1.20; 95\% CI, 0.91-1.59). Thus, decisions regarding timing of KRT initiation remain a complex issue that should be individualized based on the clinical state of the patient, in particular imminently anticipated or observed life-threatening complications of AKI.

AKI subtypes. AKI is a heterogeneous condition consisting of distinct endotypes and phenotypes based on its aetiology, prognosis and molecular pathways. Different subtypes might require and benefit from different therapeutic strategies. However, current AKI definitions based on serum creatinine and urine output provide no information on AKI subtypes. Studies using various damage and functional AKI biomarkers suggest that these new tools might overcome the limitations of current AKI definitions to improve AKI phenotyping ${ }^{106}$. Until these strategies are better embedded in clinical practice, the potential benefits of individualized therapy for AKI subtypes cannot be clearly established.

\section{Box 6 | Treatment of PO-AKI}

\section{Consensus Statement 6a}

We recommend that the underlying causes of postoperative acute kidney injury (PO-AKI) should be determined and treated whenever possible (ungraded; strong recommendation).

\section{Consensus Statement $\mathbf{6 b}$}

In patients with PO-AKI, we recommend that criteria for implementation of kidney replacement therapy (KRT) should be based on directly anticipated or observed life-threatening AKI-related complications (grade D evidence; strong recommendation).

\section{Consensus Statement 6c}

We suggest that AKI phenotype is taken into account when deciding what type of treatment to implement (ungraded; weak recommendation). 
Research recommendations. We recommend further trials to assess the optimal choice and volume of fluids for intravascular expansion and vasopressor therapy in patients with PO-AKI as well as the use of protocolbased management of haemodynamic and oxygenation parameters to prevent worsening of PO-AKI. Investigation of individual components of the KDIGO guideline to identify those that are the most effective in treating PO-AKI is important given that these were largely assembled based on expert opinion. Better understanding of AKI phenotypes is required to enable further trials to assess the response to implementation of the KDIGO bundle and other treatment options according to different AKI endotypes and phenotypes.

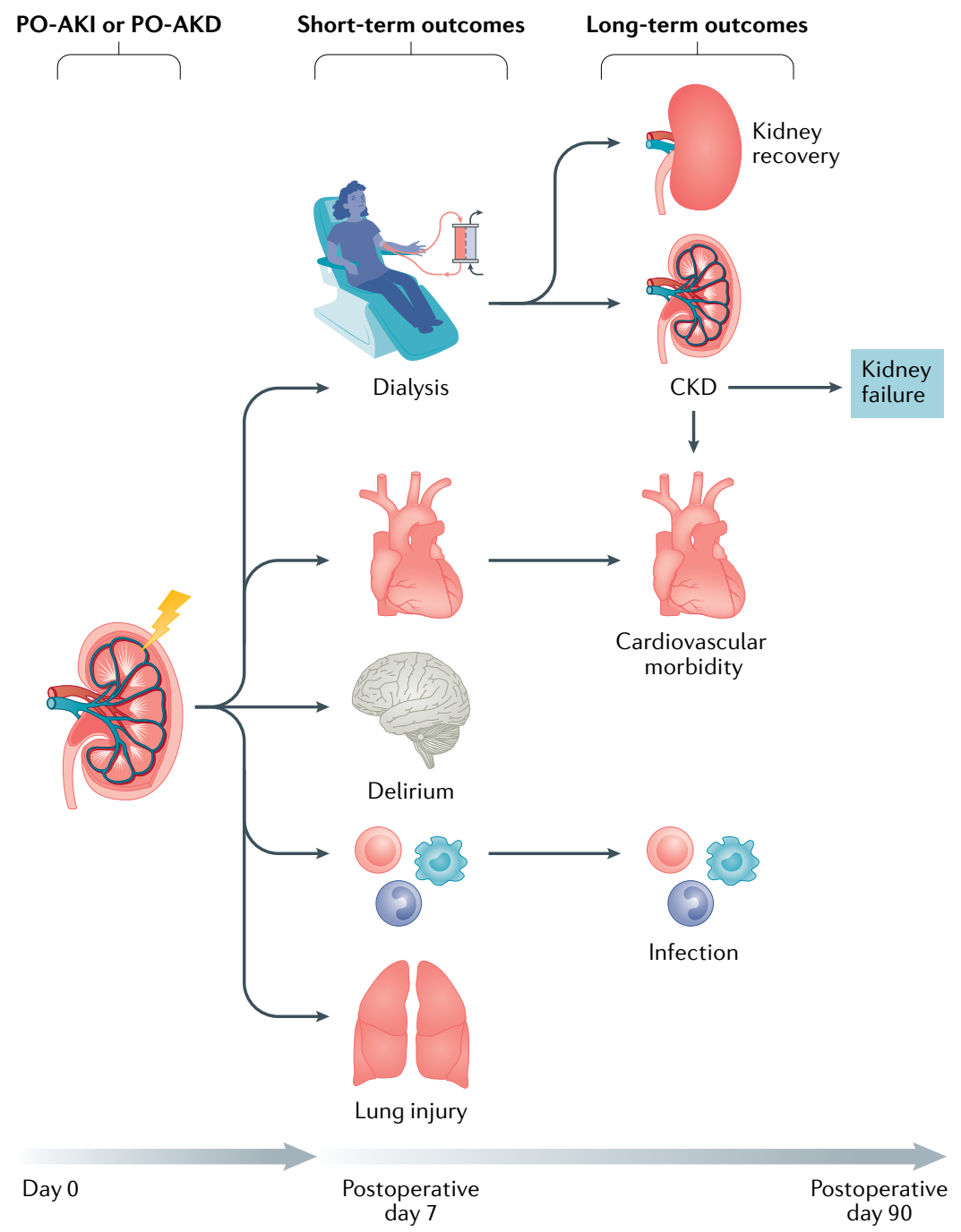

Increasing mortality, comorbidities, health-care costs, frailty, polypharmacy, drug dosing and adverse effects on management of comorbid diseases, mental health, family and friends and quality of life

Fig. 4 | Outcomes of PO-AKI and PO-AKD. Postoperative acute kidney injury (PO-AKI) is associated with an increased risk of short-term adverse outcomes, including need for dialysis, cardiovascular events, lung injury, delirium and infection. These adverse effects can in turn lead to increased long-term morbidity and mortality. Adapted from Acute Disease Quality Initiative 24, www.ADQI.org, CC BY 2.0 (https://creativecommons.org/ licenses/by/2.0/).

\section{Outcomes of postoperative AKI and AKD}

Regardless of the contributing mechanisms, PO-AKI has repeatedly been shown to be associated with a high risk of complications, including short-term and long-term mortality, high hospital costs and resource utilization $^{6,29,31,39,40,107-115}$ (FIG. 4). Data from patients admitted to an ICU for at least $24 \mathrm{~h}$ following major surgery show that those who experienced PO-AKI had persistently worse survival over 10 years, even if kidney function recovered completely ${ }^{6}$. By contrast, a retrospective analysis of patients undergoing repair of ruptured abdominal aneurysm, a very high risk of acute exposure, suggested long-term survival was not significantly different between those with and without AKI, perhaps as a result of the overwhelming influence of immediate surgical outcome in this setting ${ }^{31}$.

Patients with PO-AKI or AKD are at an increased risk of recurrent episodes of AKI and progressive deterioration of kidney function, including CKD and dialysis dependency ${ }^{39}$. In patients undergoing major surgery, the incidence of kidney failure at 1 year was $0.94 \%$ among those who experienced AKI versus $0.05 \%$ in those who did not experience $\mathrm{AKI}^{39}$. Furthermore in a competing risk model accounting for death, adjusted progression to kidney failure during 10 years of follow-up was $0.4 \%$, $2.3 \%, 7.3 \%$ and $15.7 \%$ for patients with no kidney disease, AKI with no CKD, CKD with no AKI and AKI with CKD, respectively $(P<0.001)^{39}$.

Other postoperative complications, including infection, prolonged mechanical ventilation, tracheostomy and cardiovascular events are also more common in patients who develop AKI after surgery than in those who do not ${ }^{108}$. Among 1,200 patients who underwent non-cardiac and non-vascular surgery, of whom $6.7 \%$ met the RIFLE criteria for AKI (2), the risk of non-cardiovascular complications (such as pneumonia and stroke) was similar in those with and without AKI over the first 2 weeks after surgery, but thereafter, patients with AKI experienced a significantly higher rate of cardiovascular events, including acute coronary syndrome, acute heart failure and arrhythmias ${ }^{107}$. Similarly, AKI after abdominal surgery has been associated with an increased risk of in-hospital complications, including perioperative acute myocardial infarction, pneumonia and sepsis $(P<0.001)^{29}$.

Unsurprisingly, the development of PO-AKI is associated with substantial increases in hospital costs and resource utilization, as well as longer stays in the ICU and in hospital ${ }^{29,116,117}$. Furthermore, the risks of 30-day re-admission and discharge to a nursing facility or rehabilitation centre are increased ${ }^{31}$. PO-AKI has also been associated with important, but difficult to quantify adverse outcomes, including reduced quality of life and perceived well-being ${ }^{118}$ as well as with increased risk of malignancy, possibly due to interactions with the immune system $^{119}$.

Postoperative monitoring and management. Given the increased risk of short- and long-term adverse outcomes following PO-AKI, appropriate monitoring and follow up of this cohort is a potential approach to improving patient outcomes. Although follow-up schemas for 


\begin{tabular}{|c|c|c|c|c|c|}
\hline \multicolumn{6}{|c|}{$\begin{array}{l}\text { AKI or AKD severity } \\
\text { Duration of AKI }\end{array}$} \\
\hline \multicolumn{6}{|c|}{ Recovery of kidney function } \\
\hline $\begin{array}{l}\text { - Short duration } \\
\text { of non-severe } \\
\text { AKI } \\
\text { - sCr normal or } \\
\text { returns to } \\
\text { baseline } \\
\text { - No CKD with no } \\
\text { prior } \\
\text { comorbidities }\end{array}$ & $\begin{array}{l}\text { - Short duration } \\
\text { of AKI } \\
\text { - Limited } \\
\text { preoperative } \\
\text { comorbidities } \\
\text { - No prior CKD } \\
\text { - sCr not } \\
\text { returning to } \\
\text { baseline }\end{array}$ & $\begin{array}{l}\text { - Prolonged } \\
\text { stage } 1 \mathrm{AKI} \text { or } \\
\text { brief stage } 2 \mathrm{AKI} \\
\text { - Increasing } \\
\text { comorbidities } \\
\text { and advancing } \\
\text { age } \\
\text { sCR persistently } \\
\text { elevated < } 25 \% \\
\text { above baseline }\end{array}$ & $\begin{array}{l}\text { Prolonged } \\
\text { stage } 2 \text { AKI with } \\
\text { UA showing } \\
\text { injury (duration } \\
\geq 7 \text { days) } \\
\text { Multiple } \\
\text { comorbidities } \\
\text { (cancer, prior } \\
\text { AKI, mild CKD } \\
\text { at baseline) } \\
\text { sCR persistently } \\
\text { elevated > 25\% } \\
\text { above baseline } \\
\text { but some } \\
\text { evidence of } \\
\text { recovery }\end{array}$ & $\begin{array}{l}\text { Stage } 3 \text { AKI (of } \\
\text { any duration) or } \\
\text { other persistent } \\
\text { forms of AKI or } \\
\text { kidney disease } \\
\text { - History of AKI, } \\
\text { cardiovascular } \\
\text { disease, } \\
\text { hypertension, } \\
\text { diabetes } \\
\text { mellitus and/or } \\
\text { advanced CKD }\end{array}$ & $\begin{array}{l}\text { - Dialysis- } \\
\text { requiring AKI } \\
\text { with or without } \\
\text { recovery } \\
\text { - Prior stage } 4 \\
\text { CKD } \\
\text { - Recurrent AKI } \\
\text { or AKD }\end{array}$ \\
\hline \multicolumn{6}{|c|}{ Monitoring time line } \\
\hline \multicolumn{4}{|c|}{ Within 30 days post-discharge } & \multicolumn{2}{|c|}{$2-3$ days post-discharge } \\
\hline Perioperative anc & -nephrology ca & viders & & \multicolumn{2}{|c|}{ Nephrology-based care providers } \\
\hline
\end{tabular}

Fig. 5 | Potential monitoring approach for patients who experience PO-AKI or PO-AKD. Limited data are available to inform the timing and nature of monitoring for patients who experience postoperative acute kidney injury (PO-AKI) or postoperative acute kidney disease (PO-AKD). We suggest that these patients should have their kidney function checked within 1 month of hospital discharge to confirm the extent of recovery or progression of kidney disease. Those with persistent kidney dysfunction at 90 days should be formally assessed for the development or progression of chronic kidney disease (CKD). The degree of nephrology involvement in follow-up monitoring should increase with the duration and severity of AKI or AKD commensurate with the risk of developing CKD. Patients with less severe AKI or AKD can be monitored in primary care or by the base specialist and referred for nephrology care if needed according to CKD guidelines. Future research is needed to clarify the optimal timings and methods to provide post-AKI or AKD care. Adapted from Acute Disease Quality Initiative 24, www.ADQI.org, CC BY 2.0 (https://creativecommons.org/licenses/by/2.0/).

all-cause AKI have been proposed, the optimal timing of follow-up after a postoperative episode of AKI or $\mathrm{AKD}$ is unknown ${ }^{58,120}$ and limited evidence is available to support any specific monitoring paradigm. However, adverse outcomes are increased in patients with prolonged, more severe AKI, and in those whose kidney function does not recover to baseline levels, suggesting that use of these factors to inform the initial frequency and nature of follow-up would be a reasonable approach $^{121,122}$.

Monitoring pathways should be developed in conjunction with nephrologists, but may be directly provided by perioperative physicians, primary care

\section{Box 7 | Outcomes of PO-AKI and PO-AKD}

\section{Consensus statement 7a}

The incidence of kidney and non-kidney adverse events is increased following postoperative acute kidney injury (PO-AKI) or postoperative acute kidney disease (PO-AKD). In particular, patients with surgery-associated AKI or AKD are at an increased risk of new or worsening chronic kidney disease (CKD), which is associated with increased long-term mortality (grade B evidence).

\section{Consensus Statement $\mathbf{7 b}$}

The prevention of new or worsening CKD following an episode PO-AKI or PO-AKD is a public health priority. We recommend that all patients undergo at least one kidney health assessment once the acute phase of AKI or AKD is complete. This approach will require optimal communication and AKI education between health-care professionals and the patient or caregiver (grade B evidence; strong recommendation). physicians or other specialists (FIG. 5). As a minimum, we recommend that all postoperative patients with AKI or AKD have a KHA within 30 days of hospital discharge. To facilitate this assessment, accurate information transfer from the hospital team to the primary care team and the patient or care giver is essential. At the very least, a post PO-AKI KHA should consist of checking glomerular filtration (e.g. serum creatinine levels) as well as other persistent signs of kidney injury (e.g. albuminuria). Screening for albuminuria 3 months after the development of AKI has been shown to identify those patients who are at a greater risk of CKD progression ${ }^{123}$. When available, further work-up and monitoring of kidney function with other diagnostics may be appropriate. Changes in management based on this monitoring should be communicated with the patient as well as with other treating physicians.

In survivors of $\mathrm{PO}-\mathrm{AKI}$ or $\mathrm{AKD}$, the key priorities of long-term management are to facilitate kidney recovery, prevent progressive CKD and mitigate long-term comorbidities, including cardiovascular risk. However, whether any effective strategies beyond supportive CKD care exist is unknown. ADQI recommends that a post-AKI or AKD care bundle should include assessment of kidney function, advocacy and education, review and adjustment of medications, blood pressure management and a sick day protocol ${ }^{58}$. A multidisciplinary follow-up approach (involving nephrology, 
primary care, pharmacy, a dietician, a social worker and non-nephrology subspecialists) combined with patient education is recommended but has not been formally studied. Undoubtedly, drug selection, dosing and monitoring are important and should be guided by personalized clinical decision making and regular assessment of kidney function. Both the use of nephrotoxic drugs and the inappropriate exclusion of potentially beneficial medications (for example, ACE inhibitors or antidiabetic medications) need to be avoided ${ }^{124,125}$. In patients with $\mathrm{AKD}$, in particular, a substantial risk exists of potential therapeutic failure caused by underdosing or avoidance of effective cardiovascular drugs. Close collaboration between the relevant clinical teams is paramount and future research efforts should clarify the ideal timing and method for providing post-AKI and AKD care (BOX 7).

Research recommendations. A need exists for epidemiological research to describe the frequency, nature and severity of $\mathrm{PO}-\mathrm{AKI}$ and $\mathrm{PO}-\mathrm{AKD}$ progression to CKD. The effectiveness and cost-effectiveness of supplementing a simple KHA with enhanced kidney health care in the prevention of CKD progression after surgery-associated
AKI or AKD should be evaluated in appropriately selected patients. Finally, research is needed to explore a possible common aetiology between surgery-associated $\mathrm{AKI}$ or $\mathrm{AKD}$ and other chronic organ injuries as the longer-term effects of AKI do not only involve the kidney.

\section{Conclusions}

$\mathrm{PO}-\mathrm{AKI}$ is a sentinel postoperative event that is strongly associated with both short-term surgical complications and long-term adverse outcomes. In this Consensus Statement, we provide an overview of PO-AKI and management. However, PO-AKI is a heterogeneous syndrome with a variable clinical course. In many areas the strength of evidence is poor and accordingly our recommendations are weak. We provide research recommendations for these areas to enable greater clarity to be achieved in the future. Overall, we believe that an integrated approach to $\mathrm{PO}$-AKI management requires serial evaluations of AKI risk, diagnosis, progress and outcomes throughout the perioperative period, which can be constructed as a series of focused KHAs.

\section{Published online 11 May 2021}

1. Pearse, R. M. et al. Mortality after surgery in Europe: a 7 day cohort study. Lancet 380, 1059-1065 (2012).

2. Gameiro, J., Fonseca, J. A., Neves, M., Jorge, S \& Lopes, J. A. Acute kidney injury in major abdominal surgery: incidence, risk factors, pathogenesis and outcomes. Ann. Intensive Care 8, 22 (2018).

3. Meersch, M., Schmidt, C. \& Zarbock, A. Perioperative acute kidney injury: an under-recognized problem. Anesth. Analg. 125, 1223-1232 (2017).

4. O'Connor, M. E., Kirwan, C. J., Pearse, R. M. $\&$ Prowle, J. R. Incidence and associations of acute kidney injury after major abdominal surgery. Intensive Care Med. 42, 521-530 (2016).

5. Bell, S. \& Prowle, J. Postoperative AKI-prevention is better than cure? J. Am. Soc. Nephrol. 30, 4-6 (2019).

6. Bihorac, A. et al. Long-term risk of mortality and acute kidney injury during hospitalization after major surgery. Ann. Surg. 249, 851-858 (2009)

7. Chawla, L. S., Eggers, P. W., Star, R. A. \& Kimmel, P. L. Acute kidney injury and chronic kidney disease as interconnected syndromes. N. Engl. J. Med. 371, 58-66 (2014).

8. Nadim, M. K. et al. Cardiac and vascular surgeryassociated acute kidney injury: the 20th International Consensus Conference of the ADQI (Acute Disease Quality Initiative) group. J Am. Heart Assoc. 7, e008834 (2018).

9. International Surgical Outcomes Study (ISOS) Group. Global patient outcomes after elective surgery: prospective cohort study in 27 low, middle and high income countries. Br. J. Anaesth. 117, 601-609 (2016).

10. Kellum, J. A., Bellomo, R. \& Ronco, C. Acute dialysis quality initiative (ADQI): methodology. Int. J. Artif. Organs 31, 90-93 (2008)

11. Alonso-Coello, P. et al. GRADE Evidence to Decision (EtD) frameworks: a systematic and transparent approach to making well informed healthcare choices. 1: Introduction. BMJ 353, i2016 (2016).

12. Waikar, S. S. \& McMahon, G. M. Expanding the role for kidney biopsies in acute kidney injury. Semin. Nephrol. 38, 12-20 (2018).

13. Darmon, M. et al. Diagnostic work-up and specific causes of acute kidney injury. Intensive Care Med. 43. 829-840 (2017)

14. Okusa, M. D., Rosner, M. H., Kellum, J. A. \& Ronco, C. Therapeutic targets of human AKI: harmonizing human and animal AKI. J. Am. Soc. Nephrol. 27, 44-48 (2016).

15. Bonventre, J. V. \& Yang, L. Cellular pathophysiology of ischemic acute kidney injury. J. Clin. Invest. 121, 4210-4221 (2011)

16. Linkermann, A. et al. Regulated cell death in AKI. J. Am. Soc. Nephrol. 25, 2689-2701 (2014).
17. Sutton, T. A., Fisher, C. J. \& Molitoris, B. A Microvascular endothelial injury and dysfunction during ischemic acute renal failure. Kidney Int. 62, 1539-1549 (2002).

18. Grigoryev, D. N. et al. The local and systemic inflammatory transcriptome after acute kidney injury. J. Am. Soc. Nephrol. 19, 547-558 (2008).

19. Chronopoulos, A., Cruz, D. N. \& Ronco, C. Hospitalacquired acute kidney injury in the elderly. Nat. Rev. Nephrol. 6, 141-149 (2010).

20. Kellum, J. A. \& Prowle, J. R. Paradigms of acute kidney injury in the intensive care setting. Nat. Rev. Nephrol. 14, 217-230 (2018).

21. Hoste, E. A. J. et al. Global epidemiology and outcomes of acute kidney injury. Nat. Rev. Nephrol. 14, 607-625 (2018).

22. Mcllroy, D. R. et al. Systematic review and consensus definitions for the Standardised Endpoints in Perioperative Medicine (StEP) initiative: renal endpoints. Br. J. Anaesth. 121, 1013-1024 (2018)

23. Abdullah, H. R. et al. Predictors of perioperative acute kidney injury in obese patients undergoing laparoscopic bariatric surgery: a single-centre retrospective cohort study. Obes. Surg. 26, 1493-1499 (2016).

24. Bell, S., Rennie, T., Marwick, C. A. \& Davey, P. Effects of peri-operative nonsteroidal anti-inflammatory drugs on post-operative kidney function for adults with normal kidney function. Cochrane Database Syst. Rev. 11, CD011274 (2018).

25. Bihorac, A. et al. National surgical quality improvement program underestimates the risk associated with mild and moderate postoperative acute kidney injury. Crit. Care Med. 41, 2570-2583 (2013).

26. Bonavia, A. et al. Angiotensin axis blockade, acute kidney injury, and perioperative morbidity in patients undergoing colorectal surgery: a retrospective cohort study. Medicine 98, e16872 (2019).

27. Doyle, J. F. et al. Does the implementation of a quality improvement care bundle reduce the incidence of acute kidney injury in patients undergoing emergency laparotomy? J. Clin. Med. 8, 1265 (2019).

28. Lands, V. W. et al. Reducing hypotension and acute kidney injury in the elective total joint arthroplasty population: a multi-disciplinary approach. J. Arthroplasty 33, 1686-1692 (2018).

29. Long, T. E. et al. Acute kidney injury after abdominal surgery: incidence, risk factors, and outcome. Anesth. Analg. 122, 1912-1920 (2016).

30. Rajan, S. et al. Perioperative factors associated with acute kidney injury after partial nephrectomy. Br. J. Anaesth. 116, 70-76 (2016).

31. Grams, M. E. et al. Acute kidney injury after major surgery: a retrospective analysis of veterans health administration data. Am. J. Kidney Dis. 67, 872-880 (2016).
32. Myles, P. S., Mcllroy, D. R., Bellomo, R. \& Wallace, S. Importance of intraoperative oliguria during major abdominal surgery: findings of the Restrictive versus Liberal Fluid Therapy in Major Abdominal Surgery trial. Br. J. Anaesth. 122, 726-733 (2019).

33. Shiba, A Uchino, S., Fujii, T., Takinami, M. \& Uezono, S. Association between intraoperative oliguria and acute kidney injury after major noncardiac surgery. Anesth. Analg. 127, 1229-1235 (2018).

34. Kheterpal, S. et al. Predictors of postoperative acute renal failure after noncardiac surgery in patients with previously normal renal function. Anesthesiology 107, 892-902 (2007)

35. Alpert, R. A. et al. Intraoperative urinary output does not predict postoperative renal function in patients undergoing abdominal aortic revascularization. Surgery 95, 707-711 (1984).

36. Joannidis, M. et al. Use of cell cycle arrest biomarkers in conjunction with classical markers of acute kidney injury. Crit. Care Med. 47, e820-e826 (2019).

37. Quan, S. et al. Prognostic implications of adding urine output to serum creatinine measurements for staging of acute kidney injury after major surgery: a cohort study. Nephrol Dial. Transpl. 31, 2049-2056 (2016).

38. Kellum, J. A. et al. Classifying AKI by urine output versus serum creatinine level. J. Am. Soc. Nephrol. 26 , 2231-2238 (2015).

39. Gameiro, J. et al. Acute kidney injury, long-term renal function and mortality in patients undergoing major abdominal surgery: a cohort analysis. Clin. Kidney J. 9, 192-200 (2016).

40. Zarbock, A., Koyner, J. L., Hoste, E. A. J. \& Kellum, J. A. Update on perioperative acute kidney injury. Anesth. Analg. 127, 1236-1245 (2018).

41. Grams, M. E. et al. Performance and limitations of administrative data in the identification of AKI. Clin. J. Am. Soc. Nephrol. 9, 682-689 (2014).

42. Kellum, J. A., Lameire, N. \& KDIGO AKI Guideline Work Group. Diagnosis, evaluation, and management of acute kidney injury: a KDIGO summary (Part 1). Crit. Care 17, 204 (2013).

43. Grams, M. E. et al. Candidate surrogate end points for ESRD after AKI. J. Am. Soc. Nephrol. 27, 2851-2859 (2016).

44. James, M. T. et al. Incidence and prognosis of acute kidney diseases and disorders using an integrated approach to laboratory measurements in a universal health care system. JAMA Netw. Open 2, e191795 (2019).

45. Kheterpal, S. et al. Development and validation of an acute kidney injury risk index for patients undergoing general surgery: results from a national data set. Anesthesiology 110, 505-515 (2009).

46. Bell, S. et al. Risk of postoperative acute kidney injury in patients undergoing orthopaedic surgerydevelopment and validation of a risk score and effect 
of acute kidney injury on survival: observational cohort study. BMJ 351, h5639 (2015).

47. George, L. K. et al. Association of pre-operative albuminuria with post-operative outcomes afte coronary artery bypass grafting. Sci. Rep. 5, 16458 (2015)

48. Grams, M. E. et al. A Meta-analysis of the association of estimated GFR, albuminuria, age, race, and sex with acute kidney injury. Am. J. Kidney Dis. 66, 591-601 (2015)

49. James, M. T et al. A meta-analysis of the association of estimated GFR albuminuria, diabetes mellitus, and hypertension with acute kidney injury. Am. J. Kidney Dis. 66, 602-612 (2015).

50. Wahl, T. S. et al. Association between preoperative proteinuria and postoperative acute kidney injury and readmission. JAMA Surg. 153, e182009 (2018).

51. Li, S., Wang, S., Priyanka, P. \& Kellum, J. A. Acute kidney injury in critically III patients after noncardiac major surgery: early versus late onset. Crit. Care Med. 47, e437-e444 (2019).

52. STARSurg Collaborative. Association between perioperative angiotensin-converting enzyme inhibitors and angiotensin- 2 receptor blockers and acute kidney injury in major elective non-cardiac surgery: a multicentre, prospective cohort study. Anaesthesia 73, 1214-1222 (2018)

53. Moore, P. K., Hsu, R. K. \& Liu, K. D. Management of acute kidney injury: core curriculum 2018. Am. J. Kidney Dis. 72, 136-148 (2018)

54. Kellum, J. A. et al. Kidney disease: improving globa outcomes (KDIGO) acute kidney injury work group. $\mathrm{KDICO}$ clinical practice guideline for acute kidney injury. Kidney Int. Suppl. 2, 1-138 (2012)

55. Wagener, G. et al. Urinary neutrophil gelatinaseassociated lipocalin and acute kidney injury after cardiac surgery. Am. J. Kidney Dis. 52, 425-433 (2008).

56. Stafford-Smith, M., Patel, U. D., Phillips-Bute, B. G., Shaw, A. D. \& Swaminathan, M. Acute kidney injury and chronic kidney disease after cardiac surgery. Adv. Chronic Kidney Dis. 15, 257-277 (2008).

57. Sutherland, L. et al. Acute kidney injury after cardiac surgery: a comparison of different definitions. Nephrology 25, 212-218 (2019).

58. Kashani, K. et al. Quality improvement goals for acute kidney injury. Clin. J. Am. Soc. Nephrol. 14, 941-953 (2019).

59. Bjornsson, T. D. Use of serum creatinine concentrations to determine renal function. Clin. Pharmacokinet. 4, 200-222 (1979)

60. O'Connor, M. E. et al. Acute kidney injury and mortality 1 year after major non-cardiac surgery. Br. J. Surg. 104, 868-876 (2017).

61. Chawla, L. S. et al. Acute kidney disease and renal recovery: consensus report of the Acute Disease Quality Initiative (ADOI) 16 Workgroup. Nat. Rev. Nephrol. 13, 241-257 (2017).

62. Benoit, S. W. et al. Reduction in nephrotoxic antimicrobial exposure decreases associated acute kidney injury in pediatric hematopoietic stem cell transplant patients. Biol. Blood Marrow Transpl. 25 1654-1658 (2019).

63. Bell, S. et al. Risk of AKI with gentamicin as surgical prophylaxis. J. Am. Soc. Nephrol. 25, 2625-2632 (2014).

64. Goldstein, S. L. et al. A sustained quality improvement program reduces nephrotoxic medication-associated acute kidney injury. Kidney Int 90, 212-221 (2016).

65. Stoops, C. et al. Baby NINJA (Nephrotoxic Injury Negated by Just-in-Time Action): reduction of nephrotoxic medication-associated acute kidney injury in the neonatal intensive care unit. J. Pediatr. $\mathbf{2 1 5}$ 223-228.e6 (2019).

66. Collaborative, S. T. Association between perioperative angiotensin-converting enzyme inhibitors and angiotensin- 2 receptor blockers and acute kidney injury in major elective non-cardiac surgery: a multicentre, prospective cohort study. Anaesthesia 73, 1214-1222 (2018)

67. Miller, T. E., Roche, A. M. \& Mythen, M. Fluid management and goal-directed therapy as an adjunct to Enhanced Recovery After Surgery (ERAS). Can. J. Anaesth. 62, 158-168 (2015).

68. Myles, P. S. et al. Restrictive versus liberal fluid therapy for major abdominal surgery. N. Engl. J. Med. 378, 2263-2274 (2018)

69. Grocott, M. P. et al. Perioperative increase in global blood flow to explicit defined goals and outcomes following surgery. Cochrane Database Syst. Rev. 11, CD004082 (2012).

70. Giglio, M., Dalfino, L., Puntillo, F. \& Brienza, N. Hemodynamic goal-directed therapy and postoperative kidney injury: an updated meta-analysis with trial sequential analysis. Crit. Care 23, 232 (2019).

71. Sun, L. Y., Wijeysundera, D. N., Tait, G. A. \& Beattie, W. S. Association of intraoperative hypotension with acute kidney injury after elective noncardiac surgery. Anesthesiology 123, 515-523 (2015).

72. Salmasi, V. et al. Relationship between intraoperative hypotension, defined by either reduction from baseline or absolute thresholds, and acute kidney and myocardial injury after noncardiac surgery: a retrospective cohort analysis. Anesthesiology 126, 47-65 (2017)

73. Walsh, M. et al. Relationship between intraoperative mean arterial pressure and clinical outcomes after noncardiac surgery: toward an empirical definition of hypotension. Anesthesiology 119, 507-515 (2013).

74. Calvo-Vecino, J. M. et al. Effect of goal-directed haemodynamic therapy on postoperative complications in low-moderate risk surgical patients: a multicentre randomised controlled trial (FEDORA trial). Br. J. Anaesth. 120, 734-744 (2018).

75. Asfar, P. et al. High versus low blood-pressure target in patients with septic shock. N. Engl. J. Med. 370 , 1583-1593 (2014).

76. Shaw, A. D. et al. Major complications, mortality, and resource utilization after open abdominal surgery: $0.9 \%$ saline compared to Plasma-Lyte. Ann. Surg. 255, 821-829 (2012).

77. Semler, M. W. et al. Balanced crystalloids versus saline in critically III adults. N. Engl. J. Med. 378, 829-839 (2018).

78. Self, W. H. et al. Balanced crystalloids versus saline in noncritically III adults. N. Engl. J. Med. 378, 819-828 (2018).

79. Myburgh, J. A. et al. Hydroxyethyl starch or saline for fluid resuscitation in intensive care. N. Engl. J. Med. 367, 1901-1911 (2012)

80. Perner, A. et al. Hydroxyethyl starch $130 / 0.42$ versus Ringer's acetate in severe sepsis. N. Engl. J. Med. 367, 124-134 (2012).

81. Gillies, M. A. et al. Incidence of postoperative death and acute kidney injury associated with i.v. $6 \%$ hydroxyethyl starch use: systematic review and meta-analysis. Br. J. Anaesth. 112, 25-34 (2014).

82. Kabon, B., Sessler, D. I. \& Kurz, A., Crystalloid-Colloid Study Team. Effect of intraoperative goal-directed balanced crystalloid versus colloid administration on major postoperative morbidity: a randomized trial. Anesthesiology 130, 728-744 (2019).

83. Futier, E. et al. Effect of hydroxyethyl starch vs saline for volume replacement therapy on death or postoperative complications among high-risk patients undergoing major abdominal surgery: the FLASH randomized clinical trial. JAMA 323, 225-236 (2020).

84. Sessler, D. I. et al. Period-dependent associations between hypotension during and for four days after noncardiac surgery and a composite of myocardial infarction and death: a substudy of the POISE-2 Trial. Anesthesiology 128, 317-327 (2018).

85. Futier, E. et al. Effect of Individualized vs standard blood pressure management strategies on postoperative organ dysfunction among high-risk patients undergoing major surgery: a randomized clinical trial. JAMA 318, 1346-1357 (2017).

86. Frisch, A. et al. Prevalence and clinical outcome of hyperglycemia in the perioperative period in noncardiac surgery. Diabetes Care 33, 1783-1788 (2010).

87. Mudumbai, S. C. et al. Thirty-day mortality risk associated with the postoperative nonresumption of angiotensin-converting enzyme inhibitors: a retrospective study of the Veterans Affairs Healthcare System. J. Hosp. Med. 9, 289-296 (2014).

88. Lee, S. M., Takemoto, S. \& Wallace, A. W. Association between withholding angiotensin receptor blockers in the early postoperative period and 30-day mortality: a cohort study of the veterans affairs healthcare system. Anesthesiology 123, 288-306 (2015).

89. Marcotte, J. H. et al. Acute kidney injury following implementation of an enhanced recovery after surgery (ERAS) protocol in colorectal surgery. Int. J. Colorectal Dis. 33, 1259-1267 (2018)

90. Schunk, S. J. et al. Association between urinary dickkopf-3, acute kidney injury, and subsequent loss of kidney function in patients undergoing cardiac surgery: an observational cohort study. Lancet 394 488-496 (2019)

91. Hollinger, A. et al. Proenkephalin A 119-159 (Penkid) is an early biomarker of septic acute kidney injury: the kidney in sepsis and septic shock (Kid-SSS) Study. Kidney Int. Rep. 3, 1424-1433 (2018).

92. Koyner, J. L. et al. Tissue inhibitor metalloproteinase-2 (TIMP-2)IGF-binding protein-7 (IGFBP7) levels are associated with adverse long-term outcomes in patients with AKI. J. Am. Soc. Nephrol. 26 1747-1754 (2015).

93. Kashani, K. et al. Discovery and validation of cell cycle arrest biomarkers in human acute kidney injury. Crit. Care 17, R25 (2013).

94. Greenberg, J. H. et al. Biomarkers of AKI progression after pediatric cardiac surgery. J. Am. Soc. Nephrol. 29, 1549-1556 (2018).

95. Parikh, C. R. et al. Postoperative biomarkers predict acute kidney injury and poor outcomes after adult cardiac surgery. J. Am. Soc. Nephrol. 22, 1748-1757 (2011).

96. Meersch, M. et al. Prevention of cardiac surgeryassociated AKI by implementing the KDIGO guidelines in high risk patients identified by biomarkers: the PrevAKI randomized controlled trial. Intensive Care Med. 43, 1551-1561 (2017)

97. Gocze, I. et al. Biomarker-guided intervention to prevent acute kidney injury after major surgery: the prospective randomized BigpAK study. Ann. Surg. 267, 1013-1020 (2018).

98. Hoste, E. A. et al. RIFLE criteria for acute kidney injury are associated with hospital mortality in critically ill patients: a cohort analysis. Crit. Care 10, R73 (2006)

99. Uchino, S., Bellomo, R., Goldsmith, D., Bates, S. $\&$ Ronco, C. An assessment of the RIFLE criteria for acute renal failure in hospitalized patients. Crit. Care Med. 34, 1913-1917 (2006).

100. Ricci, Z., Cruz, D. \& Ronco, C. The RIFLE criteria and mortality in acute kidney injury: A systematic review. Kidney Int. 73, 538-546 (2008).

101. Joannidis, M. et al. Acute kidney injury in critically ill patients classified by AKIN versus RIFLE using the SAPS 3 database. Intensive Care Med. 35 1692-1702 (2009).

102. Ali, T. et al. Incidence and outcomes in acute kidney injury: a comprehensive population-based study. J. Am. Soc. Nephrol. 18, 1292-1298 (2007).

103. Zarbock, A. et al. Effect of early vs delayed initiation of renal replacement therapy on mortality in critically ill patients with acute kidney injury: the ELAIN randomized clinical trial. JAMA 315, 2190-2199 (2016).

104. Gaudry, S. et al. Initiation strategies for renalreplacement therapy in the intensive care unit. N. Engl. J. Med. 375, 122-133 (2016).

105. Bagshaw, S. M. et al. Timing of initiation of renalreplacement therapy in acute kidney injury. N. Engl. J. Med. 383, 240-251 (2020).

106. Huen, S. C. \& Parikh, C. R. Molecular phenotyping of clinical AKI with novel urinary biomarkers. Am. J. Physiol. Renal Physiol. 309, F406-F413 (2015).

107. Biteker, M. et al. Incidence, risk factors, and outcomes of perioperative acute kidney injury in noncardiac and nonvascular surgery. Am. J. Surg. 207, 53-59 (2014).

108. Hobson, C., Lysak, N., Huber, M., Scali, S. \& Bihorac, A. Epidemiology, outcomes, and management of acute kidney injury in the vascular surgery patient. J. Vasc. Surg. 68, 916-928 (2018).

109. Kopolovic, I. et al. Risk factors and outcomes associated with acute kidney injury following ruptured abdominal aortic aneurysm. BMC Nephrol. 14, 99 (2013).

110. Arora, P. et al. The effect of acute kidney injury after revascularization on the development of chronic kidney disease and mortality in patients with chronic limb ischemia. J. Vasc. Surg. 61, 720-727 (2015).

111. Drews, J. D., Patel, H. J., Williams, D. M., Dasika, N. L $\&$ Deeb, G. M. The impact of acute renal failure on early and late outcomes after thoracic aortic endovascular repair. Ann. Thorac. Surg. 97, 2027-2033; discussion 2033 (2014).

112. Ozrazgat-Baslanti, T. et al. Acute and chronic kidney disease and cardiovascular mortality after major surgery. Ann. Surg. 264, 987-996 (2016).

113. Jalalzadeh, $\mathrm{H}$. et al. Long-term survival after acute kidney injury following ruptured abdominal aortic aneurysm repair. J. Vasc. Surg. 66, 1712-1718.e2 (2017).

114. Ko, T. et al. Impact of acute kidney injury on early to long-term outcomes in patients who underwent surgery for type a acute aortic dissection. Am. J. Cardiol. 116, 463-468 (2015).

115. Bravi, C. A. et al. Impact of acute kidney injury and its duration on long-term renal function after partial nephrectomy. Eur. Urol. 76, 398-403 (2019).

116. Romagnoli, S. et al. Postoperative acute kidney injury in high-risk patients undergoing major abdominal surgery. J. Crit. Care 35, 120-125 (2016).

117. Konda, P. et al. Identification of risk factors associated with postoperative acute kidney injury after 
esophagectomy for esophageal cancer. J. Cardiothorac. Vasc. Anesth. 31, 474-481 (2017).

118. Porter, A. C. et al. Predictors and outcomes of healthrelated quality of life in adults with CKD. Clin. J. Am. Soc. Nephrol. 11, 1154-1162 (2016).

119. Chao, C. T. et al. Dialysis-requiring acute kidney injury increases risk of long-term malignancy: a populationbased study. J. Cancer Res. Clin. Oncol. 140 613-621 (2014).

120. Silver, S. A. \& Siew, E. D. Follow-up care in acute kidney injury: lost in transition. Adv. Chronic Kidney Dis. 24, 246-252 (2017).

121. Mehta, S. et al. The prognostic importance of duration of AKI: a systematic review and meta-analysis. BMC Nephrol. 19, 91 (2018).

122. See, E. J. et al. Long-term risk of adverse outcomes after acute kidney injury: a systematic review and metaanalysis of cohort studies using consensus definitions of exposure. Kidney Int. 95, 160-172 (2019).

123. Hsu, C. Y. et al. Post-acute kidney injury proteinuria and subsequent kidney disease progression: the assessment, serial evaluation, and subsequent sequelae in acute kidney Injury (ASSESS-AKI) Study. JAMA Intern. Med. 180, 402-410 (2020).

124. Ostermann, M. et al. Drug management in acute kidney disease - report of the acute disease quality initiative XVI meeting. Br. J. Clin. Pharmacol. 84, 396-403 (2018)

125. Brar, S. et al. Association of angiotensin-converting enzyme inhibitor or angiotensin receptor blocker use with outcomes after acute kidney injury. JAMA Intern. Med. 178, 1681-1690 (2018).

Author contributions

J.R.P., L.G.F., M.S.C., M.E., M.E.G., M.P.W.G., K.D.L., D.M., P.T.M., M.O., A.Z. and S.M.B. wrote the text. All authors researched the data for the article and reviewed or edited the manuscript before submission.

\section{Competing interests}

The Acute Disease Quality Initiative (ADQI)-24 and the PeriOperative Quality Initiative (POOI)-7 Conference was supported by unrestricted education grants from the following companies: Baxter Inc, B. Braun Melsungen, BioMérieux SA AG, Cytosorbents Inc, Edwards Lifesciences Inc, La Jolla Pharmaceutical Inc, MediBeacon Inc, Medtronic Inc and Trevena Inc. A.Z. has received consulting and/or lecture fees from Astute Medical/BioMerieux, Fresenius and Baxter. A.Z. has received grant support from Astute Medical/BioMerieux, Fresenius and Baxter. R.M.P. has held research grants and has given lectures and/or performed consultancy work for Intersurgical, GlaxoSmithKline and Edwards Lifesciences, and holds editorial roles with the British Journal of Anaesthesia, the British Journal of Surgery and BMJ Quality and Safety. M.B. reports research funding from Baxter Inc. M.G.M. is a consultant for Edwards Lifesciences and co-inventor of a clinical hydration device (CliniQuench Ltd). A.B. was supported by NIH Research Project Grant Program R01 GM110240. T.E.M reports research funding and is a consultant for Edwards Lifesciences. S.M.B. reports receiving fees for scientific advisory and speaking for Baxter, for scientific advisory for CNA Diagnostics, for study clinical adjudication for BioPorto, and for travel from Spectral Medical. S.M.B. is sup ported by a Canada Research Chair in Critical Care Nephrology. T.J.G. reports honoraria from Acacia, Edwards, Medtronic and Merck. J.L.K. reports research funds from Astute Medical, Nxstage Medical, NIH, Satellite Healthcare and consulting Fees from Astute Medical, Baxter, Sphingotec. P.T.M has advisory board memberships with FAST Biomedical, AM-Pharma, Sphingotec. M.J. reports honoraria and research support from Baxter Healthcare Corp, AM-Pharma, CLS Behring, Fresenius and Astute Medical. M.S.C. reports honoraria from B Braun and Edwards Lifesciences and sits on the Advisory Board for Edwards Lifesciences. J.A.K. has received grant/research support from Astellas, Astute Medical, Baxter, bioMérieux, Cytosorbents RenalSense, consulting fees from Astellas, Astute Medical, Baxter, bioMérieux, Cytosorbents, RenalSense, DaVita, Fresenius, Jafron, Mallinckrodt, NxStage, Potrero, and has licensing of intellectual property for Astute Medical and Cytosorbents. M.O. declared having received consultancy fees from NxStage, speaker honoraria from Fresenius Medica Care and research support from LaJolla Pharma. K.D.L. declared having received consultancy fees from bioMérieux, speaker honoraria from Baxter, and stock options from Amgen. J.R.P. declared having received consultancy fees from MediBeacon, Nikkiso Europe $\mathrm{GmbH}$, and Quark Pharmaceuticals; speaker honoraria from Baxter, Fresenius Medical Care, and Nikkiso Europe $\mathrm{GmbH}$; and research support from bioMérieux. A.D.S. acts as a consultant for Edwards Lifesciences, FAST Biomedical and Astellas Pharma. L.G.F. has received honoraria and research support from Astute Medical, La Jolla Pharmaceuticals, Medibeacon, Baxter and Fresenius. The other authors declare no competing interests.

\section{Peer review information}

Nature Reviews Nephrology thanks Prasad Devarajan, Jose Lopes and the other, anonymous, reviewer(s) for their contribution to the peer review of this work.

\section{Publisher's note}

Springer Nature remains neutral with regard to jurisdictional claims in published maps and institutional affiliations.

\section{Supplementary information}

The online version contains supplementary material available at https://doi.org/10.1038/s41581-021-00418-2.

\section{(c) (i)}

Open Access This article is licensed under a Creative Commons Attribution 4.0 International License, which permits use, sharing, adaptation, distribution and reproduction in any medium or format, as long as you give appropriate credit to the original author(s) and the source, provide a link to the Creative Commons license, and indicate if changes were made. The images or other third party material in this article are included in the article's Creative Commons license, unless indicated otherwise in a credit line to the material. If material is not included in the article's Creative Commons license and your intended use is not permitted by statutory regulation or exceeds the permitted use, you will need to obtain permission directly from the copyright holder. To view a copy of this license, visit http://creativecommons.org/licenses/by/4.0/.

(c) The Author(s) 2021 